\title{
Lípidos en el anfípodo Talorchestia margaritae (Amphipoda: Talitridae) y su relación con la ecología de la especie
}

\author{
Sandra López ${ }^{1}$, Yusbelly Díaz ${ }^{1}$, Karem Noris ${ }^{2} \&$ Aivle Cabrera ${ }^{3}$ \\ 1. Laboratorio de Crustáceos Peracáridos. Departamento de Estudios Ambientales. Universidad Simón Bolívar, A.P. \\ 89000, Caracas 1080, Venezuela; sslopezpereney@gmail.com, yusdiaz@usb.ve \\ 2. Departamento de Biología Celular. Universidad Simón Bolívar, A.P. 89000; Caracas 1080, Venezuela; \\ knoris@usb.ve \\ 3. Departamento de Química, Universidad Simón Bolívar, A.P. 89000, Caracas 1080, Venezuela; \\ aivlecabrera@gmail.com
}

Recibido 16-VIII-2009. Corregido 16-III-2010. Aceptado 20-IV-2010.

\begin{abstract}
Lipids in the amphipod Talorchestia margaritae (Amphipoda: Talitridae) and its relationship with the ecology of the species. T. margaritae, an endemic species inhabiting Venezuelan coasts, plays an important ecological role in plant and animal decomposition. To understand this issue in some animal groups, especially small ones, lipid composition analysis has been an interesting tool to describe their trophic relationships and food preferences. In order to assess this and visualize the components of their diet, we determined the lipid composition differences between males and females and among age classes in this species. Two sandy beaches were selected: Mangle Quemao and Las Mercedes de Paparo, from which sand samples of known volume were collected at the supralittoral area in 2007. Organisms were separated by age and sex classes, and their size, weight, density, biomass, total lipids (TL), lipid classes and fatty acid markers present in their tissues were determined. The sizes were similar for all age classes between the two locations, while the weights were higher for Mangle Quemao. The TL and lipid classes showed similar proportions between sexes, age classes and locations (TL: 3-5\%; Phospholipids: 20-30\%; Glycolipids: $<1 \%$; sterols: $4 \%$ ). On the other hand, Triglycerides (TAG) were higher in Mangle Quemao, which may be related to the difference between the weights of two locations. The most abundant fatty acid biomarkers in the two studied sites were 16:0 and 18:1(n-9); this last one is characteristic of a carnivorous diet. The other nine markers were identified with changes in their distribution in organisms at Mangle Quemao and between males and females of both populations. Based on observed fatty acids markers we can assume T. margaritae as a generalist carnivore. Those populations were influenced by available food; inducing differences in weight, TAG proportion and markers diversity. Rev. Biol. Trop. 58 (3): 841-855. Epub 2010 September 01.
\end{abstract}

Key words: talitrid, fatty acid biomarkers, diet, amphipod, sandy beaches, Venezuela, community structure.

Talorchestia margaritae (Stephensen 1948) es un anfípodo gammárido perteneciente a la familia Talitridae que fue descrito por primera vez en la isla de Margarita por Stephensen (1948). Esta especie, endémica de Venezuela ha sido ampliamente estudiada en cuanto a su biología y ecología (Blanco 1980, Venables 1981 a,b,c, Sánchez 1985). Su distribución geográfica abarca las costas desde la región oriental hasta la central donde comienza a aparecer T. marcuzzi (Rufo 1954), organismo que predomina en las costas occidentales del país (Lemos 2002). T. margaritae pertenece al grupo de los anfípodos semiterrestres, que se localiza en el área húmeda de la línea supralitoral. Blanco (1980), Venables (1981a,b,c) y Sánchez (1985) han evaluado sus parámetros biológicos con fines de cultivo, debido a que la fracción asimilable de esta especie y su producción se encuentra entre los máximos valores 
señalados, al compararlos con los de otras especies animales.

Este anfípodo cumple un rol importante en la descomposición de los restos vegetales depositados en las playas y presenta altas densidades cuando están disponibles abundantes cantidades de alimento, alcanzando hasta 39 047.00ind. $/ \mathrm{m}^{2}$ (Venables $1981 \mathrm{a}, \mathrm{b}$ ). Basado en una dieta sólo a base de Thalassia sp., principal componente alimenticio reportado para la especie, el consumo de alimento diario de los jóvenes es de casi un $40 \%$ de su peso, descendiendo de forma considerable en los adultos (Sánchez 1985). Cuando la población está dominada por individuos adultos, la fracción de energía asimilada por la especie que se representa en la relación producción/[respiración + producción] tiende a disminuir en sus valores desde un $60 \%$ hasta un $40 \%$ (Venables 1981a, Sánchez 1985).

Correa (1985) determinó que existe un incremento calórico significativo entre los jóvenes $(4.86 \mathrm{kcal} / \mathrm{g}$ peso seco sin ceniza) y los adultos (machos y hembras) de la especie, mientras que no existen diferencias calorimétricas significativas entre los machos adultos $(5.38 \mathrm{kcal} / \mathrm{g}$ peso seco sin ceniza) y las hembras no ovígeras ( $5.13 \mathrm{kcal} / \mathrm{g}$ peso seco sin ceniza). Las hembras ovígeras (hembras+embrión $=6.68 \mathrm{kcal} / \mathrm{g}$ peso seco sin ceniza) presentan valores significativamente mayores a los machos adultos y a las hembras no ovígeras, siendo la diferencia con este último grupo de $1.55 \mathrm{kcal} / \mathrm{g}$ peso seco $\mathrm{sin}$ ceniza. Esta diferencia, se puede deber a la acumulación de nutrientes en los tejidos por parte de la hembra para la producción de huevos, o a que los huevos también aportan valores calorimétricos. Estos resultados conllevaron a la autora a sugerir la realización de estudios que determinen la concentración de lípidos, proteínas y carbohidratos en los diferentes tejidos de T. margaritae, con el objetivo de completar el perfil energético de la especie.

Los lípidos han recibido especial atención en el área de la ecología, debido a sus cadenas ricas en componentes carbonados, que representan un gran valor energético en los sistemas marinos, siendo los ácidos grasos los mayores contribuyentes en los extractos lipídicos (Parrish et al. 2000). Su importancia radica en que son la mínima expresión de las moléculas lipídicas más complejas luego de la digestión, siendo integrados intactos a los tejidos (Iverson et al. 2004, Nyssen 2005), por lo que es posible diferenciarlos entre aquellos que son provenientes o no de la dieta, ya que sólo un número limitado de ácidos grasos son sintetizados de novo (Iverson et al. 2004). Esto permite determinar las relaciones tróficas y las preferencias alimenticias de diferentes grupos animales, y en el caso de los anfípodos, evita el tener que realizar análisis del contenido del tracto digestivo (Mayzaud \& Ackman 1976, Graeve et al. 1997, Bühring \& Christiansen 2001, Auel et al. 2002), ya que estos organismos pueden adaptar su composición lipídica a las condiciones nutricionales, tanto a nivel de los lípidos de reserva como de sus membranas celulares, siendo mínimas las diferencias entre los fosfolípidos y los triacilgliceroles (Graeve et al. 2001).

El objetivo del presente estudio fue determinar los lípidos totales, las clases de lípidos y el contenido de marcadores de ácidos grasos presentes en individuos de $T$. margaritae por sexo y categorías de talla, en dos playas arenosas en la región centro-oriental de Venezuela, con el fin de determinar así la posible posición trófica de la especie en condiciones naturales.

\section{MATERIALES Y MÉTODOS}

Área de muestreo: Los anfípodos de la especie T. margaritae fueron recolectados en dos playas arenosas de la costa centro-oriental venezolana. Durante el mes de agosto de 2007, estos organismos se recolectaron en la localidad Mangle Quemao (10²3'20” N, 64²4'10” E), bahía protegida ubicada en el Parque Nacional Mochima, estado Sucre y dominada por praderas de Thalassia testudinum Banks ex König 1805. En septiembre del mismo año, la recolecta se llevó a cabo en las Mercedes de Paparo $\left(10^{\circ} 22^{\prime} 39.5^{\prime \prime} \mathrm{N}, 65^{\circ} 58^{\prime} 16.6^{\prime \prime}\right)$, playa arenosa en el estado Miranda, la cual a diferencia de Mangle Quemao posee una gran extensión y 
deposición de sedimentos debido a las descargas del río Tuy.

Parámetros poblacionales: Para determinar la densidad y biomasa de la especie T. margaritae en ambas playas, se tomaron muestras en seis puntos (tres replicas en cada uno) separadas a cinco metros a lo largo de la zona supralitoral, utilizando un cilindro ( $8.5 \mathrm{~cm} x 4.5 \mathrm{~cm}$ de diámetro). Se recolectaron un total de 103 individuos en Mangle Quemao y 202 en las Mercedes de Paparo. Posteriormente en el laboratorio fueron separados por sexo gracias al dimorfismo sexual del gnatópodo dos (macho con gnatópodo bien desarrollado $\mathrm{y}$ el de las hembras atrofiado) y por tallas en: jóvenes $(1.00-3.90 \mathrm{~mm})$, ejemplares inmaduros $(4.00-7.90 \mathrm{~mm})$ y ejemplares maduros (8.00-12.00mm), midiendo la longitud total del organismo desde el extremo de la antena dos al telson. La diferencia entre inmaduros y adultos se estableció por el grado de desarrollo del gnatópodo dos de los machos y extrapolando esta talla al grupo de las hembras (Venables 1981c, Lemos 2002). Las hembras maduras se separaron en ovígeras y no ovígeras. Todos los organismos fueron pesados (peso húmedo) en una balanza Sartorius $(0.0000 \mathrm{mg})$. Con los datos obtenidos se hicieron correlaciones lineales entre las tallas y los pesos húmedos para establecer la distribución de los datos y estimar las diferencias poblacionales entre ambas localidades para cada una de las categorías de talla mediante pruebas no-paramétricas de Kruskal-Wallis (95\% de confianza), luego de ser probada la condición de no normalidad de los datos con las pruebas Anderson-Darling $(\mathrm{p}<0.005)$.

Análisis de lípidos: Paralelamente se colectaron organismos vivos de la especie a lo largo de la zona supralitoral evaluada para los parámetros poblaciones, sacudiendo el material orgánico depositado en bandejas con agua de mar fría, para inducir su letargo. Los ejemplares de T. margaritae fueron separados en las diferentes categorías de talla y sexo, hasta obtener $1 \mathrm{~g}$ aproximado de muestra por cada una y por replica, obteniendo $\mathrm{n}$ de muestras variables para cada caso, debido a la dependencia de la densidad y biomasa disponible en cada localidad. A un grupo de las hembras ovígeras les fueron extraídos los huevos del marsupio, para cuantificar el aporte lipídico de los mismos a la hembra. Finalmente, los organismos frescos fueron preservados en nitrógeno líquido hasta el momento de los análisis. Los lípidos totales (LT) fueron extraídos con una solución de cloroformo - metanol 2:1 (v/v) y determinados por gravimetría, para ser expresados como LT/ mg. Los esteroles y triglicéridos (TAG) fueron determinados mediante kit enzimáticos comerciales de los Laboratorios Wiener Lab $\subset$, mientras que los fosfolípidos (FL) se cuantificaron empleando el método optimizado de Zhou \& Arthur (1992). Para el análisis de los glicolípidos se siguió el método descrito por Kates (1972). Los resultados fueron expresados como porcentaje de esteroles, FL, glicolípidos y TAG respecto a los lípidos totales.

Se determinó la presencia de algunos marcadores de ácidos grasos, tanto en los organismos de estudio, para determinar los diferentes componentes de su dieta, así como a muestras de sedimento provenientes de cada localidad, con el fin de identificar la biodisponibilidad de estos en el detritos del sedimento, siguiendo el método descrito en Jaffé et al. (1995). Los análisis de ácidos grasos se hicieron con un cromatógrafo de gases marca Agilent modelo 7890 acoplado a un detector selectivo de masas modelo 5975C. La cuantificación se realizó mediante la determinación del área relativa de los picos de los siguientes marcadores de ácidos grasos saturados: 15:0, 16:0, 17:0 y los insaturados 16:1(n-7), 18:1(n-9), 18:2(n6), 18:3(n-3), 18:4(n-3), 20:1(n-9), 20:5(n-3), $22: 1(n-11)$ y $22: 6(n-3)$. Debido a que no se observaron diferencias en la distribución entre los ejemplares inmaduros o maduros en los perfiles de ácidos grasos obtenidos para las diferentes categorías, en los posteriores análisis se utilizaron las categorías de macho (M), donde fueron incluidos los machos maduros e inmaduros; hembras $(\mathrm{H})$, con las hembras maduras, inmaduras y ovígeras; y los jóvenes (J). Los 
resultados obtenidos de los ácidos grasos contenidos en cada una de las muestras de ambas localidades fueron comparados empleando la prueba $\chi^{2}$ (Chi-cuadrado, 95\% de confianza).

\section{RESULTADOS}

Parámetros poblacionales: En ambas poblaciones se observó una relación de tipo exponencial entre el peso húmedo $(\mathrm{mg})$ de los individuos y su talla $(\mathrm{mm})$, presentando un $\mathrm{R}^{2}$ de 0.90 para Mangle Quemao $\left(y=0.0593 x^{2.4304}\right) \mathrm{y}^{2}$ de 0.91 para las Mercedes de Paparo $\left(\mathrm{y}=0.0122 \mathrm{x}^{3.0169}\right)$. Las hembras ovígeras y no ovígeras (hembras maduras), hembras inmaduras y machos maduros de T. margaritae no presentaron diferencias significativas en las tallas medias entre las dos localidades de estudio, sin embargo, si se encontraron diferencias significativas entre las tallas de los jóvenes y machos inmaduros (Kruskal-Wallis, $\mathrm{p}<0.05$ ). Con relación al peso húmedo, todas las categorías de individuos de la localidad de Mangle Quemao, presentaron un peso superior respecto a los individuos de las Mercedes de Paparo. Así mismo, el peso medio para las hembras maduras y las hembras ovígeras no presentó diferencias significativas, mientras que los jóvenes, los machos, las hembras inmaduras y los machos inmaduros sí las presentaron (Kruskal-Wallis, $\mathrm{p}<0.05$ ) (Cuadro 1).

La densidad promedio de T. margaritae en las Mercedes de Paparo fue aproximadamente tres veces mayor (2 367ind. $/ \mathrm{m}^{2}$ ) que en Mangle Quemao (855ind. $\left./ \mathrm{m}^{2}\right)$, mientras que la biomasa solamente se duplicó. A nivel de categorías, los jóvenes presentaron la mayor densidad, siendo su proporción mayor al 70\% de la densidad total en ambas localidades, mientras que los restantes grupos presentaron proporciones menores al 20\%, donde los maduros de ambos sexos fueron los que presentaron las proporciones más bajas $(<4 \%)$, observándose una tendencia a la disminución del número de individuos en la medida en que las tallas aumentan (Fig. 1A). En lo que respecta a la biomasa de individuos por clase, todas estas aportan aproximadamente el mismo porcentaje de peso $(20 \%)$ a la biomasa total para las dos

CUADRO 1

Talla y peso promedio de T. margaritae por categoría. Valores promedio $\pm D S$

TABLE 1

Average size and weight of T. margaritae by class. Average values $+S D$

\begin{tabular}{|c|c|c|c|c|c|c|}
\hline \multirow{2}{*}{ Categoría } & \multicolumn{3}{|c|}{ Mangle Quemao } & \multicolumn{3}{|c|}{ Las Mercedes de Paparo } \\
\hline & $\mathrm{n}$ & Talla (mm) & Peso (mg) & $\mathrm{n}$ & Talla (mm) & Peso (mg) \\
\hline Macho maduro $* \mathrm{P}$ & 5 & $\begin{array}{c}8.98 \pm 1.00 \\
(8.00-10.00)\end{array}$ & $\begin{array}{c}11.62+4.76 \\
(7.44-18.93)\end{array}$ & 22 & $\begin{array}{c}8.73 \pm 0.83 \\
(8.00-11.00)\end{array}$ & $\begin{array}{c}7.75 \pm 1.90 \\
(4.82-12.66)\end{array}$ \\
\hline Macho inmaduro*T.P & 6 & $\begin{array}{c}6.67 \pm 0.61 \\
(6.00-7.50)\end{array}$ & $\begin{array}{c}6.74 \pm 1.12 \\
(5.67-8.89)\end{array}$ & 32 & $\begin{array}{c}5.91 \pm 0.85 \\
(4.00-7.50)\end{array}$ & $\begin{array}{c}3.65 \pm 1.27 \\
(1.30-6.40)\end{array}$ \\
\hline Hembra madura & 5 & $\begin{array}{c}8.40 \pm 0.55 \\
(7.00-9.00)\end{array}$ & $\begin{array}{c}9.63 \pm 1.41 \\
(6.39-11.52)\end{array}$ & 19 & $\begin{array}{c}8.32 \pm 0.58 \\
(8.00-10.00)\end{array}$ & $\begin{array}{c}7.94+1.79 \\
(5.84-12.51)\end{array}$ \\
\hline Hembra inmadura*P & 32 & $\begin{array}{c}5.05 \pm 0.95 \\
(4.00-7.00)\end{array}$ & $\begin{array}{c}2.97 \pm 1.72 \\
(1.48-9.83)\end{array}$ & 79 & $\begin{array}{c}5.07 \pm 1.12 \\
(4.00-7.50)\end{array}$ & $\begin{array}{c}2.42 \pm 1.76 \\
(0.73-6.27)\end{array}$ \\
\hline Jóvenes*T.P & 134 & $\begin{array}{c}2.30 \pm 0.61 \\
(1.00-3.50)\end{array}$ & $\begin{array}{c}0.61 \pm 0.52 \\
(0.10-1.78)\end{array}$ & 352 & $\begin{array}{c}2.03 \pm 0.63 \\
(1.00-3.90)\end{array}$ & $\begin{array}{c}0.13 \pm 0.17 \\
(0.12-1.20)\end{array}$ \\
\hline Hembra ovígera & 3 & $\begin{array}{c}8.00 \pm 0.00 \\
(8.00)\end{array}$ & $\begin{array}{c}8.20 \pm 0.78 \\
(7.60-9.08)\end{array}$ & 13 & $\begin{array}{c}7.95 \pm 0.94 \\
(7.00-10.00)\end{array}$ & $\begin{array}{c}7.09 \pm 2.09 \\
(5.17-11.32)\end{array}$ \\
\hline
\end{tabular}

1 n: número de individuos por clase/number of individuals per class

2 ( ) valores mínimo y máximo reportado/minimum and maximum values reported

3 * diferencias significativas ( $\mathrm{p}<0.05)$; P: peso; T: talla/significant differences $(\mathrm{p}<0.05)$, P: weight, T: size. 


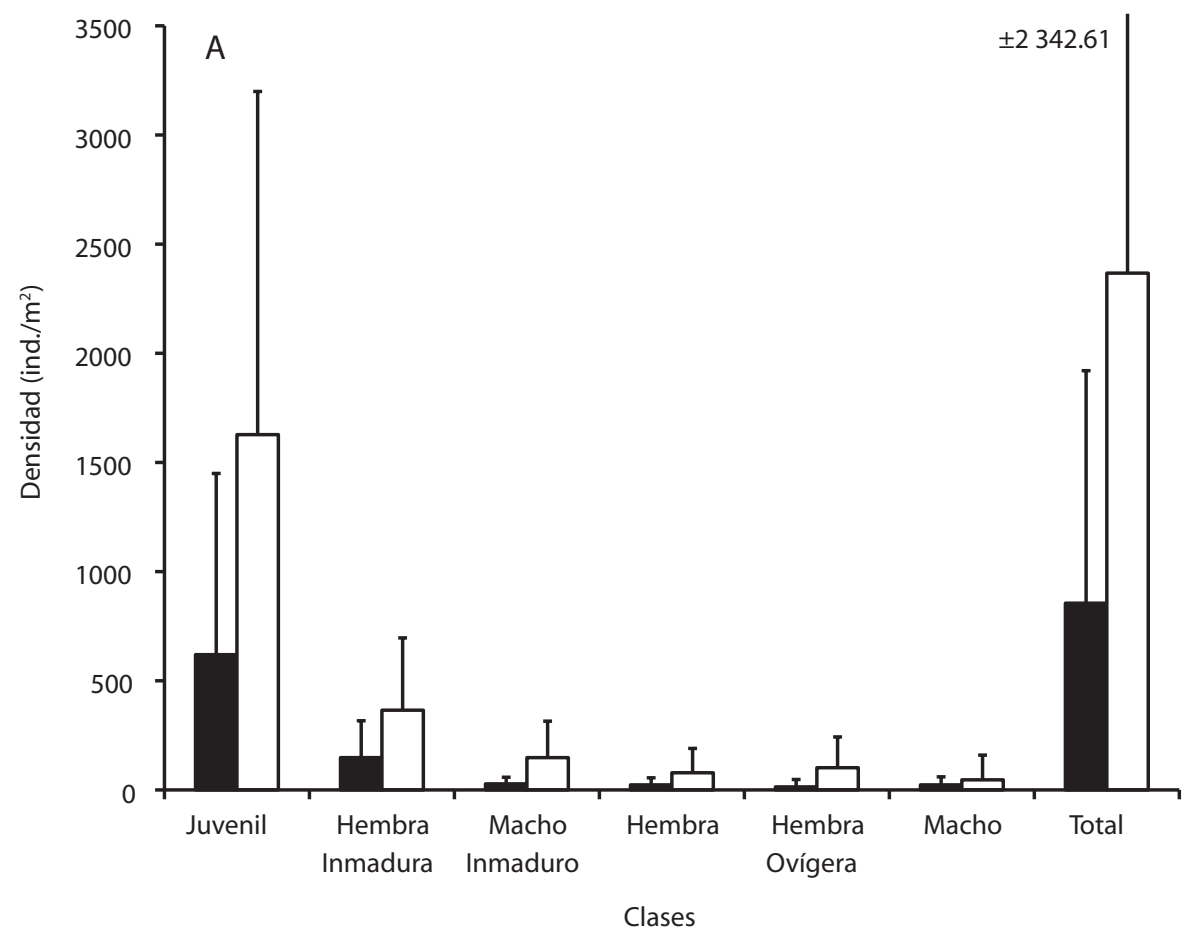

- Mangle Quemao $\square$ Las Mercedes de Paparo

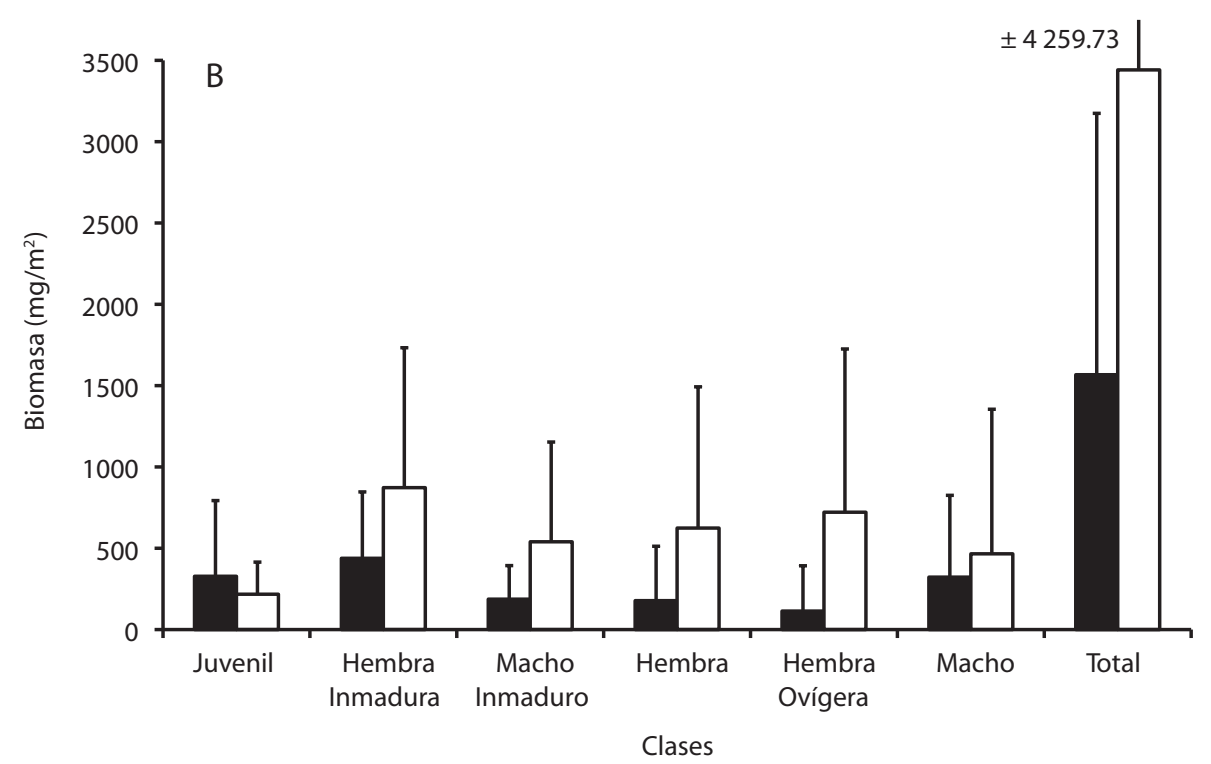

- Mangle Quemao $\square$ Las Mercedes de Paparo

Fig. 1. Densidad (A) y Biomasa (B) de organismos por categoría y totales en las dos localidades de estudio.

Fig. 1. Density (A) and biomass (B) of organisms per class and total in the two localities. 


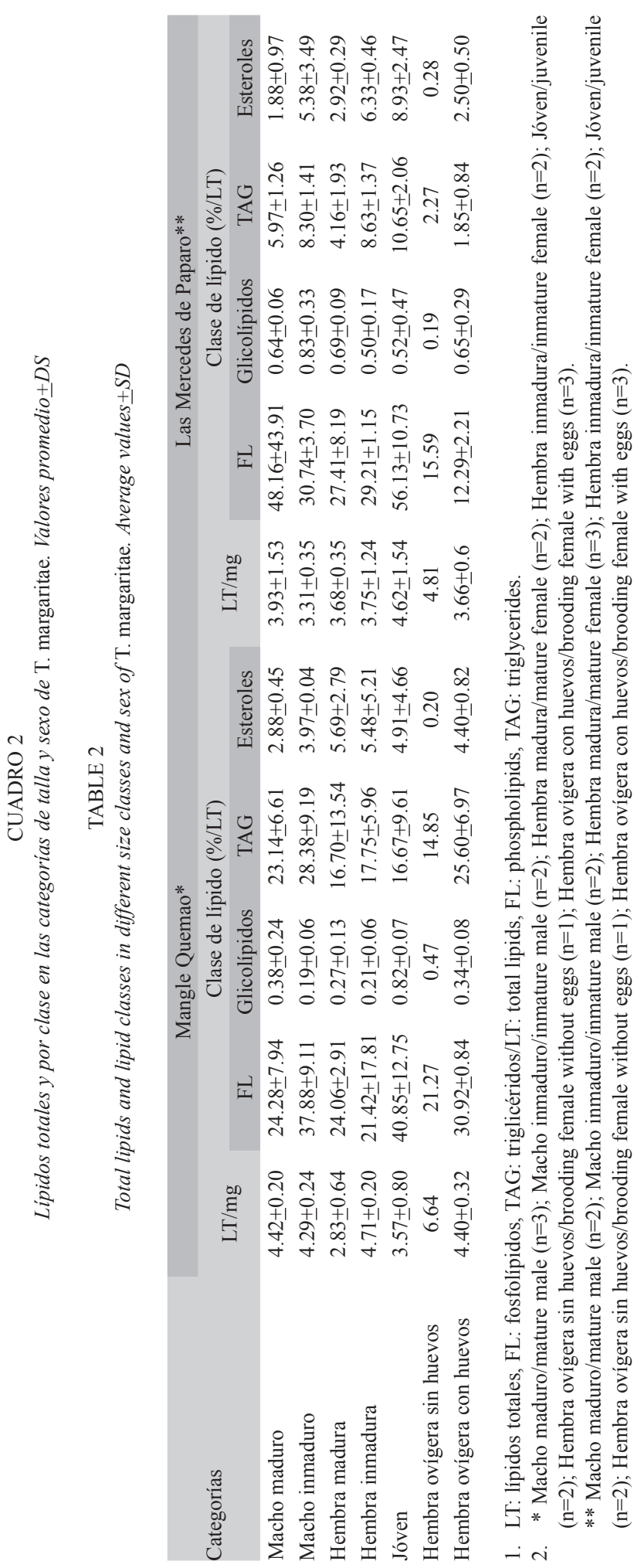


localidades, no observándose ningún patrón asociado entre los valores y la biomasa de organismos con su talla (Fig. 1B).

Análisis de lípidos: En las dos localidades de estudio la proporción de LT es similar para todas las categorías de tallas y sexo, sin embargo en la localidad de Mangle Quemao, las hembras maduras contenían un $66 \%$ menos de LT, mientras que las hembras ovígeras a las cuales les fueron extraídos sus huevos contenían $55 \%$ más que el promedio del resto de las categorías. Los lípidos más abundantes en las dos localidades fueron los FL, seguidos en orden de importancia por los TAG, los esteroles y los glicolípidos. Todas las clases de lípidos presentaron proporciones similares entre los diferentes grupos evaluados en las dos localidades, con la excepción de los TAG, los cuales se observan en menores proporciones en las Mercedes de Paparo, con respecto a Mangle Quemao (Cuadro 2).

Igualmente, se puede observar que los LT del grupo de las hembras ovígeras a las cuales les fueron extraídos sus huevos son un $31 \%$ y $50 \%$ mayor que los LT de las hembras ovígeras con sus huevos de las Mercedes de Paparo y Mangle Quemao, respectivamente. Por otra parte, esta última conserva una proporción de esteroles similar a la de las hembras no ovígeras, encontrándose esta proporción drásticamente reducida en las hembras ovígeras sin sus huevos (Cuadro 2).

De los 12 ácidos grasos evaluados en este estudio sólo los 15:0, 16:0, 16:1(n-7), 17:0, 18:1(n-9) y 18:2(n-6) fueron encontrados en todas las categorías de tallas y sexo, siendo el 16:0 y el 18:1(n-9) los más abundantes en las dos localidades y en todos los grupos, independientemente del sexo. Otros cuatro marcadores fueron encontrados distribuidos entre las diferentes categorías para la localidad de Mangle Quemao 20:1(n-9), 20:5(n-3), 22:1(n11) y 22:6(n-3) y tres para las Mercedes de Paparo 20:1(n-9), 22:1(n-11) y 22:6(n-3). Los biomarcadores $18: 3(n-3)$ y $18: 4(n-3)$ no fueron detectados en ninguna de las dos localidades, mientras que el 20:5(n-3) no fue detectado en las Mercedes de Paparo.

Siguiendo la nueva distribución de las categorías propuesta en la metodología para estos análisis, se obtuvo que en Mangle Quemao se encontraron diferencias significativas en la distribución de los marcadores entre las diferentes categorías evaluadas $\left(\chi^{2}, \mathrm{p}<0.001\right)$. En los tres grupos de esta localidad se observa la presencia de los marcadores saturados 15:0 y 17:0, así como los polinsaturados 18:1(n-9), 20:1(n-9) y 22:1(n-11), siendo el más abundante el ácido graso 18:1(n-9). En menos abundancia, se observaron los marcadores 16:1(n-7), 18:2(n6), 20:5(n-3) y 22:6(n-3) (Fig. 2A), mientras que los marcadores $18: 3(n-3)$ y $18: 4(n-3)$ no fueron detectados en el análisis.

Por otra parte, en las Mercedes de Paparo, no se observaron diferencias significativas entre los diferentes componentes de la dieta para las tres categorías evaluadas $\left(\chi^{2}, \mathrm{p}>0.05\right)$, siendo más uniforme la distribución de los marcadores de ácidos grasos en esta localidad. En las tres categorías se observaron los marcadores 15:0, 16:1(n-7), 17:0, 18:1(n-9), 18:2(n-6) y 20:1(n-9), mientras que los marcadores de ácidos grasos 22:1(n-11) y 22:6(n-3), sólo fueron detectados en las hembras y en los jóvenes en muy bajas proporciones (Fig. 2B).

Al realizar el análisis en relación con las categorías de organismos, se observó que en los machos de ambas playas, el marcador más abundante fue 18:1(n-9). El resto de los marcadores presentaron áreas menores al 10\%, con la excepción del 18:2(n-6), el cual aporta un 28\% aproximadamente en la dieta de los machos en la localidad de Mangle Quemao. La distribución de los marcadores en los machos presentó diferencias significativas entre sí $\left(\chi^{2}, p<0.001\right)$, observándose mayor diversidad de éstos en los machos de Mangle Quemao (Fig. 3A).

Las hembras presentaron una distribución de marcadores similar a la de los machos, donde el más abundante en ambas localidades es 18:1(n-9), mientras que los demás marcadores presentaron áreas menores al $10 \%$. La distribución de los marcadores entre las hembras de ambas localidades presentó diferencias 

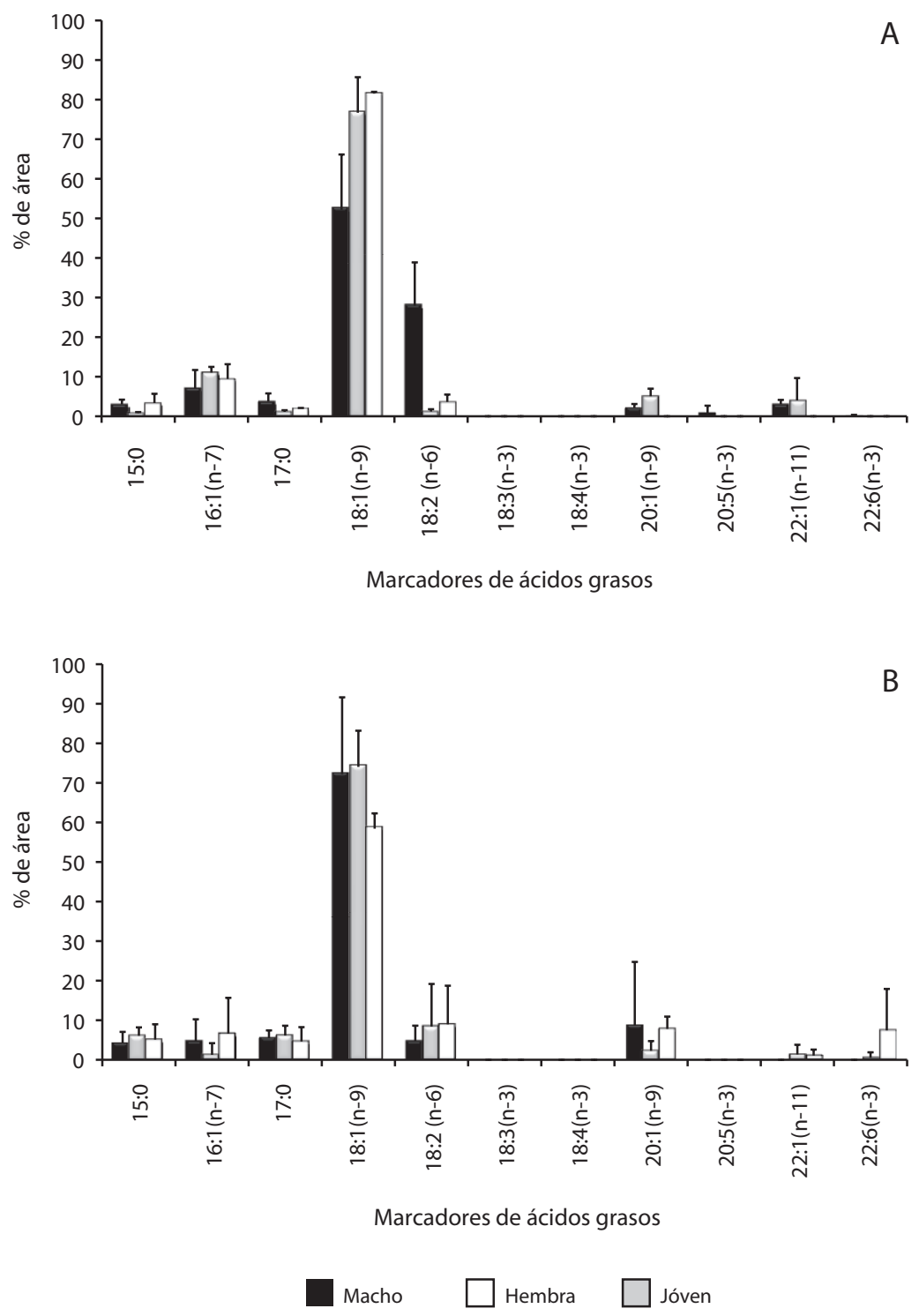

Fig. 2. Porcentaje de los marcadores de ácidos grasos evaluados en (A) Mangle Quemao. (B) Las Mercedes de Paparo. Promedio y desviación estándar.

Fig. 2. Percentage of fatty acid markers evaluated in (A) Mangle Quemao. (B) Las Mercedes de Paparo. Mean and standard deviation.

significativas al compararlas entre sí $\left(\chi^{2}\right.$, $\mathrm{p}<0.001$ ) (Fig. 3B). Por último, los jóvenes de las dos localidades no presentaron diferencias significativas en lo que respecta a la distribución de estos biomarcadores $\left(\chi^{2}, \mathrm{p}>0.05\right)$. $\mathrm{Al}$ igual que en las hembras y los machos de ambas localidades, en los jóvenes, el marcador más abundante encontrado fue el ácido graso 18:1(n-9) (Fig. 3C).

Con el fin de determinar los marcadores de ácidos grasos biodisponibles en el detritos del sedimento, se determinaron sus ácidos 

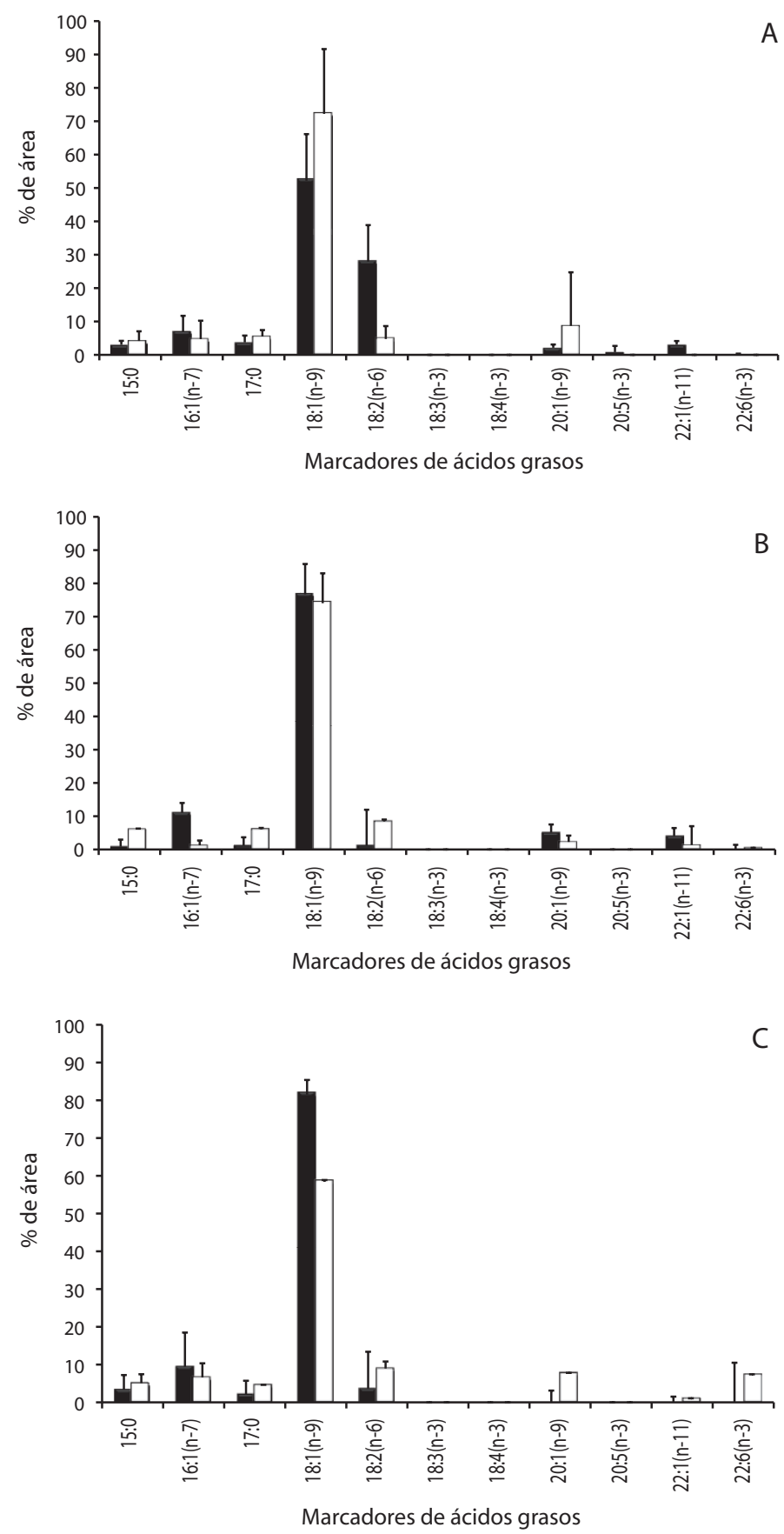

Mangle Quemao $\square$ Las Mercedes de Paparo

Fig. 3. Porcentaje de los marcadores de ácidos grasos evaluados en (A) machos, (B) hembras y (C) jóvenes de las dos localidades. Promedio y desviación estándar.

Fig. 3. Percentage of fatty acid markers evaluated in (A) male, (B) female and (C) juveniles of the two localities. Mean and standard deviation. 
presentes, encontrándose un total de seis marcadores en Mangle Quemao 15:0, 16:0, 17:0, 18:1(n-9), 20:1(n-9) y 22:1(n-11), mientras que en las Mercedes de Paparo sólo se detectaron cuatro 15:0, 16:0, 17:0 y 18:1(n-9) en tan sólo una de las tres muestras evaluadas. En ambas localidades el ácido graso más importante fue el 18:1(n-9), con $45.14 \pm 8.63 \%$ para Mangle Quemao y $51.92 \%$ para las Mercedes de Paparo. El segundo en importancia fue el ácido graso saturado $16: 0$, con $41.57 \pm 8.80 \%$ y $44.85 \%$ respectivamente, mientras que los demás biomarcadores detectados presentaron abundancias menores al 6\%. Los marcadores 16:1(n-7), 18:2(n-6), 18:3(n-3), 18:4(n-3), $20: 5(n-3)$ y $22: 6(n-3)$ no fueron detectados (Cuadro 3).

CUADRO 3

Marcadores de ácidos grasos presentes en el sedimento. Valores promedio $\pm D S$

TABLE 3

Fatty acids markers present in the sediment. Average values $\pm S D$

$\begin{array}{lcc}\text { Ácidos grasos } & \begin{array}{c}\text { Mangle Quemao } \\ \%(\mathrm{n}=3)\end{array} & \begin{array}{c}\text { las Mercedes } \\ \text { de Paparo } \\ \%(\mathrm{n}=1)\end{array} \\ 15: 0 & 1.09 \pm 0.14 & 1.72 \\ 16: 0 & 41.57 \pm 8.80 & 44.85 \\ 17: 0 & 1.36 \pm 0.22 & 1.51 \\ 18: 1(\mathrm{n}-9) & 45.14 \pm 8.63 & 51.92 \\ 20: 1(\mathrm{n}-9) & 5.84 \pm 2.49 & * * \\ 22: 1(\mathrm{n}-11) & 5.01 \pm 2.89 & * *\end{array}$

1 ** Por debajo del rango de detección del método o no detectado/below the method detection range or not detected.

\section{DISCUSIÓN}

Utilizando la clasificación de tallas propuesta por Lemos (2002) para establecer las categorías etarias, se pudo observar que para los individuos inmaduros y adultos existe un aumento en el peso húmedo promedio, respecto al reportado. Esta autora establece que los individuos pequeños o jóvenes están entre los
$0.26 \mathrm{mg}$ a $0.84 \mathrm{mg}, 2.32 \mathrm{mg}$ para los medianos o inmaduros y $3.23 \mathrm{mg}$ para los grandes o maduros. Venables (1981b) reporta una relación lineal entre estos dos parámetros para esta especie en la costa de Cumaná, con $\mathrm{R}^{2}=0.98$, a diferencia de lo que ocurre en los organismos de las dos localidades de estudio. Los datos reportados en el presente estudio sugieren que existe un aumento en los pesos de los organismos, posiblemente debido a un aumento en la disponibilidad de alimento en las diferentes localidades de estudio. Por otra parte, Sánchez (1985) estableció que las diferentes categorías de tallas consumen en promedio entre $12.19 \%$ y $39.90 \%$ de alimento en una dieta solamente a base de Thalassia sp., donde los organismos de tallas más grandes, consumen menos que los de tallas pequeñas con relación a su peso.

Todos los estudios apuntan a que el éxito reproductivo y energético de esta especie se encuentra asociado a la disponibilidad de alimento, específicamente de Thalassia testudinum, muy común en la costa oriental de Venezuela, como Cumaná, isla de Margarita y en una de las localidades de estudio, Mangle Quemao (Blanco 1980, Venables 1981a,b,c, Correa 1985, Sánchez 1985, Lemos 2002). Sin embargo, en las Mercedes de Paparo, se presentan lo más altos valores de densidad y biomasa para T. margaritae, aún cuando su principal alimento no se encuentra presente. Este resultado sugiere que $T$. margaritae es generalista y que en la región oriental de Venezuela ingiere mayoritariamente la fanerógama por su gran abundancia. Sin embargo, por observaciones de campo hechas sobre la especie, esta es capaz de aprovechar otros componentes menos abundantes, pero disponibles en su hábitat, posiblemente traídos por las corrientes marinas.

Los lípidos son la mayor fuente de energía de los organismos y representan uno de los principales componentes en el peso total de los mismos, esto se debe a los triglicéridos y a los fosfolípidos. Los primeros corresponden a las moléculas de reservas alimenticias y los segundos a los componentes celulares estructurales (Parrish et al. 2000, Nelson \& Cox 2005, Fenucci \& Harán 2006). Correa 
(1985) cuantificó el contenido calórico de algunos estadíos del desarrollo y sexo para T. margaritae, observando que en promedio todas las categorías de talla y sexo conservan un contenido calórico similar, sin diferencias significativas, exceptuando a las hembras ovígeras, las cuales presentaron la mayor tasa metabólica. Esto se refleja igualmente en los lípidos totales y en las clases de lípidos para las dos localidades evaluadas en este estudio, donde los porcentajes de LT se conservan en proporciones similares entre todas las categorías de talla y sexo evaluadas, como ocurre en la especie dulceacuícola Hyalella curvispina Shoemaker 1942 la cual acumula lípidos en proporción a la oferta estacional que tiene de alimento y no presentan diferencias importantes en la cantidad de lípidos entre sexos (Poretti et al. 2003). En ese estudio no se encontraron resultados concluyentes en lo que respecta a las hembras ovígeras, al igual que en nuestro caso con las hembras ovígeras de T. margaritae a las cuales les fueron extraídos sus huevos, que presentaron un mayor valor de LT, incluso que las hembras ovígeras analizadas con sus huevos presentes.

Esta diferencia entre las hembras ovígeras analizadas con sus huevos y sin ellos, se puede deber a que estos porcentajes son estandarizados con base en el peso de los organismos, por lo que los huevos aportan peso a la hembra ovígera pero no lípidos. Los huevos de esta especie son de crecimiento rápido y se encuentran libres dentro del marsupio de la madre una vez que ésta los deposita ahí luego de su fecundación (Huck et al. 2007). El período de gestación completo dura aproximadamente entre seis y siete días (Blanco 1980) y el consumo de otras biomoléculas como las proteínas se reducen en un $50 \%$ aproximadamente en este período (Huck et al. 2007); por lo que no es raro esperar que los lípidos, la principal fuente de energía de consumo directo en los huevos, tenga una tasa de consumo igual o mayor, tal y como ocurre en otros anfípodos como Gammarus oceanicus (Segerstråle 1947) y Echinogammarus marinus (Leach 1815) (Clarke et al. 1985).
Los principales lípidos integrados a los huevos de las hembras en el presente estudio parecen ser los esteroles. Sin embargo, no existe otra evidencia que pueda confirmar esta hipótesis, debido a que en este estudio no se contó con réplicas para esta última categoría y en otras publicaciones, tales como Clarke et al. (1985), en las cuales se miden los lípidos a diferentes estadíos de huevos en las especies de anfípodos G. oceanicus y E. marinus, donde no se observa esta tendencia.

En lo que respecta a las clases de lípidos, las proporciones de los cuatro tipos evaluados no varían entre las diferentes categorías de talla y sexo, así como para las dos localidades de estudio, con la excepción de los TAG, donde los organismos de las Mercedes de Paparo almacenan muy pocos en comparación con los de Mangle Quemao. Estas variaciones coinciden con las diferencias de pesos en las categorías de talla y sexo entre las dos localidades, donde este siempre es menor en las Mercedes de Paparo. Esto se puede deber, probablemente, a la ausencia de la fanerógama en esta localidad, la cual ha sido reportada como una gran fuente de energía y proteínas (Sánchez 1985).

En las dos localidades se observa que los ácidos grasos más abundantes en las diferentes categorías de talla y sexo fueron el 16:0 y 18:1(n-9). Estos ácidos han sido previamente reportados como los más abundantes en varias especies de gammáridos, caprélidos e hipéridos (Scott et al. 2001, Nelson et al. 2001, Auel et al. 2002, Correia et al. 2003, Guerra-García et al. 2004). La composición de ácidos grasos en los organismos de las dos localidades es similar, diferenciándose entre sí en las proporciones en las cuales se encontraron, así como en las categorías de talla y sexo en donde se observaron. Por este motivo, fueron reordenadas las categorías y se evaluaron las diferencias intra e inter poblacionales de la especie. Los marcadores de ácidos grasos presentes en todas las categorías en las dos localidades fueron los ácidos grasos 15:0 y 17:0, los cuales determinan la presencia de bacterias anaeróbicas (Parrish et al. 2000). El 16:1(n-7) representante del grupo de las diatomeas (Graeve et al. 1997, 2001, Parrish et 
al. 2000, Bühring \& Christiansen 2001, Nelson et al. 2001, Scott et al. 2001, Auel et al. 2002, Guerra-García et al. 2004), el 18:1(n-9), característico de la carnivoría (Auel et al. 2002), siendo este el más bioacumulado en los tejidos de $T$. margaritae y el 18:2(n-6), marcador de plantas terrestres (Parrish et al. 2000).

La mayor abundancia del marcador 18:1(n9) en las dos localidades de estudio, evidencia una dieta carnívora, la cual es bastante similar en lo que respecta a la composición de marcadores presentes a una carroñera, variando la preferencia de lo vivo por lo muerto, tal y como ocurre entre las especies Waldeckia obesa Chevreux 1905 (necrófago), Eusirus perdentatus Chevreux 1912 (carnívoro-macrodepredador) y Echiniphimedia hodgsoni Walker 1906 (carnívoro-microdepredador) (Grave et al. 2001). Sin embargo, el importante rol descrito para esta especie en la región oriental durante el proceso de descomposición de los restos vegetales depositados en las playas, especialmente de Thalassia sp., podría sugerir que esta especie es detritívora, en lo que respecta al menos a Mangle Quemao, tal y como ocurre en la especie Epimeria georgiana Schellenberg 1931, la cual ha sido reportada como detritívora, pero su composición de ácidos grasos refleja una importante proporción del marcador 18:1(n-9), al igual que otros marcadores vegetales (Grave et al. 2001). Sin embargo, en la localidad de las Mercedes de Paparo, no existe evidencia o reportes de la actividad de esta especie sobre los restos vegetales presentes en esta localidad.

Otros marcadores encontrados en los tejidos de T. margaritae, fueron los ácidos grasos 20:1(n-9), 20:5(n-3), 22:1(n-11) y 22:6(n-3). Estos marcadores representan igualmente una ingesta de base carnívora de organismos más específicos como lo son los copépodos, el zooplancton en general, las macroalgas/flagelados (Graeve et al. 1997, 2001; Parrish et al. 2000, Bühring \& Christiansen 2001, Nelson et al. 2001, Scott et al. 2001, Auel et al. 2002, Guerra-García et al. 2004). Estos resultados concuerdan con la hipótesis de que esta especie es generalista y que su ingesta depende de su capacidad digestiva, para procesar los diferentes componentes, debido a que en otras especies de talítridos como Talorchestia sp., T. marmorata (Haswell) y Keratroides vulgaris (Friend 1979), Johnston et al. (2005), se reportaron diferencias en la capacidad digestiva de los mismos, dependiendo de las preferencias alimenticias de cada especie, evaluando la relación entre las enzimas digestivas que producían y las biomoléculas que predominaban en su dieta.

Evaluando la biodisponibilidad en cada una de las localidades de estudio se observó que la diversidad de marcadores en el sedimento es baja, siendo los más abundantes los ácidos grasos 16:0 y el 18:1(n-9), lo que sugiere una gran cantidad de restos animales en la materia orgánica presente. Otros marcadores observados en bajas proporciones fueron los marcadores de las bacterias anaeróbicas (15:0 y 17:0) en las dos localidades y sólo en Mangle Quemao con un 5\% los marcadores de copépodos y zooplancton en general.

Las diferencias en los componentes de la dieta entre tallas y sexos observadas en Mangle Quemao, se ha reportado para otras especies de anfípodos donde las hembras y los machos presentan una composición de ácidos grasos significativamente diferentes entre sí, tal y como ocurre en las especies de caprélidos como Caprella acanthifera Leach 1814, C. danilevskii Czerniavski 1868 y C. penantis Leach 1814 (Guerra-García et al. 2004), así como entre los jóvenes, sub-adultos, hembras adultas y machos adultos del gammárido Gammarus locusta Linnaeus 1758 (Correia et al. 2003).

Estos resultados, pueden reflejar diferencias en las estrategias alimenticias entre las categorías de talla y sexo dentro de una misma localidad, ya sea, posiblemente, por un aumento de la talla con mayor eficiencia, o como estrategia reproductiva entre machos y hembras (Correia et al. 2003, Guerra-García et al. 2004). Lo que puede significar que las poblaciones de T. margaritae de la localidad de las Mercedes de Paparo poseen una menor diversidad de fuentes de alimento aprovechables para esta especie, debido a la poca variabilidad observada en la distribución de estos componentes. 
La diferencia significativa existente en los componentes de la dieta entre los machos de ambas localidades se puede deber a la variación espacial de las dos poblaciones y los recursos alimenticios disponibles. Esto, puede también explicar las diferencias de la dieta en las hembras. Casos similares han sido reportados, entre otros, por Auel et al. (2002), quienes establecieron que el hipérido Themisto abyssorum (Boeck 1870), presentaba una gran concentración de marcadores de carnivoría en el estrecho de Fram, mientras que en el Océano Ártico central presentaba una gran cantidad del marcador 16:1(n-7), característico de una dieta a base de diatomeas.

Por otro lado, Guerra-García et al. (2004) reportaron que en tres especies de caprélidos, C. acanthifera, C. penantis y Pseudoprotella phasma (Montagu 1804) variaba la proporción de los marcadores 16:1(n-7) - diatomeas y 22:6(n-3) - flagelados, entre las tres localidades de estudio, estableciéndoles a cada localidad, un nivel de impacto antropogénico, donde las diatomeas aumentaban su proporción desde la localidad menos impactada a la más impactada y los flagelados a la inversa. Se establece que estos resultados se debieron a cambios en los niveles de eutrofización entre las localidades o los cambios en la temperatura del agua entre las tres localidades.

El análisis de ácidos grasos aporta información útil respecto a los diferentes componentes de la dieta, aparte de Thalassia sp., en poblaciones naturales de $T$. margarita. Igualmente, aporta concomiendo sobre las necesidades nutricionales para cada una de las categorías de tallas, el cual puede ser utilizado para la cría y mantenimiento en condiciones de laboratorio, partiendo del hecho que esta especie puede habitar en localidades donde no se encuentra la fanerógama.

En estudios previos realizados en las dos localidades evaluadas en el presente trabajo, se han reportado diferencias en el comportamiento de $T$. margaritae ante agentes estresantes, tales como el grado de canibalismo y diferencias en las proporciones de categorías de tallas y sexo (Lemos 2002). Así mismo, es evidente la influencia que deben tener las descargas del río Tuy en la localidad de las Mercedes de Paparo, aportando grandes cantidades de desechos de origen antropogénico, por lo que las dos localidades pueden proporcionar componentes alimenticios diferentes.

\section{AGRADECIMIENTOS}

Agradecemos a Alberto Martín, Andrés Martín e Ileana Ortega por el apoyo logístico en la recolección del material biológico y a Igor Meléndez por su colaboración en el montaje de los diferentes ensayos. Igualmente agradecemos a los revisores por sus acertados comentarios para el mejoramiento del material presentado. Este proyecto fue parcialmente financiado por el Decanato de Estudios Profesionales de la Universidad Simón Bolívar.

\section{RESUMEN}

T. margaritae cumple un papel importante en la descomposición de restos vegetales y animales en las costas venezolanas. El objetivo fue determinar las diferencias en la composición lipídica entre sexos y talla de la especie. Para esto, especímenes de T. margaritae fueron recolectados en la zona supralitoral de dos playas arenosas: Mangle Quemao y las Mercedes de Paparo. Posteriormente se separaron por talla y sexo determinándoles su peso, densidad, biomasa y perfil lipídico. Todas las categorías presentaron tallas similares entre localidades. Los pesos fueron superiores en los organismos de Mangle Quemao. Los lípidos totales presentaron proporciones similares entre sexos, talla y localidad (3-5\%), al igual que los fosfololípidos (20-30\%), glicolípidos $(<1 \%)$ y esteroles $(4 \%)$, exceptuando los triglicéridos (TAG), siendo estos superiores en Mangle Quemao. Esto último pudiendo estar relacionado con la diferencia de pesos entre localidades. Los ácidos grasos más abundantes en ambas localidades fueron 16:0 y 18:1(n-9). Se identificaron otros 9 marcadores con variaciones en su distribución. Con base en los biomarcadores se puede catalogar a T. margaritae como una especie carnívora-generalista, siendo sus poblaciones influenciadas por la disponibilidad de alimento que a su vez, podría inducir diferencias de peso, TAG y biomarcadores.

Palabras clave: talítrido, marcadores ácidos grasos, dieta, anfípodo, playas arenosas, Venezuela, estructura poblacional. 


\section{REFERENCIAS}

Auel, H., M. Harjes, R. da Rocha, D. Stübing \& W. Hagen. 2002. Lipid biomarkers indicate different ecological niches and trophic relationships of the Arctic hyperiid amphipods Themisto abyssorum and T. libellula. Polar Biol. 25: 374-383.

Blanco, J. 1980. Contribución al conocimiento de la biología de Talorchestia margaritae Stephensen, 1948 (Amphipoda: Talitridae). Tesis de Licenciatura, Universidad de Oriente, Cumaná, Venezuela.

Bühring, S. \& B. Christiansen. 2001. Lipids in selected abyssal benthopelagic animals links to the epipelagic zone? Prog. Oceanogr. 50: 369-384.

Clarke, A., A. Skadsheim \& L. Holmes. 1985. Lipid biochemistry and reproductive biology in two species of Gammaridae (Crustacea: Amphipoda). Mar. Biol. 88: $247-263$.

Correa, M. 1985. Estimación del contenido calórico de Talorchestia margaritae (Talitridae: Amphipoda). Bol. Inst. Oceanogr. 24: 11-14.

Correia, A., M. Costa, O. Luis \& D. Livingstone. 2003. Age-related changes in antioxidant enzyme activities, fatty acid composition and lipid peroxidation in whole body Gammarus locusta (Crustacea: Amphipoda). J. Exp. Mar. Biol. Ecol. 289: 83-101.

Fenucci, J. \& N. Harán. 2006. Acción de los lípidos en crustáceos penaeoideos, p. 1-31. In C. Rosas, O. Carrillo, R. Wilson \& E. Andreatta (eds.). Estado Actual y perspectivas de la nutrición de los camarones peneidos cultivados en Iberoamérica. CYTED, Mexico D.F., Mexico.

Graeve, M., G. Kattner \& D. Piepenburg. 1997. Lipids in Arctic benthos: does the fatty acid and alcohol composition reflect feeding and trophic interactions? Polar Biol. 18: 53-61.

Graeve, M., P. Dauby \& Y. Scailteur. 2001. Combined lipid, fatty acid and digestive tract content analyses: a penetrating approach to estimate feeding modes of Antarctic amphipods. Polar Biol. 24: 853-862.

Guerra-García, J., I. Martínez-Pita \& M. Pita. 2004. Fatty acid composition of the Caprellidea (Crustacea: Amphipoda) from the Strait of Gibraltar. Sci. Mar. 68: 501-510.

Huck, E., A. Martín \& P. Miloslavich. 2007. Contenido proteico en los diferentes estadíos de embriones de Talorchestia margaritae Stephensen, 1948
(Amphipoda, Talitridae) durante su desarrollo intramarsupio. Rev. Biol. Trop. 55: 1-8.

Iverson, S., C. Field, W. Don Bowen \& W. Blanchard. 2004. Quantitative fatty acid signature analysis: a new method of estimating predator diets. Ecol. Monographs 74: 211-235.

Jaffé, R., G. Wolff, A. Cabrera \& H. Carvajal-Chitty. 1995. The biogeochemistry of lipids in rivers of the Orinoco Basin. Geochim. Cosmochim. Ac. 59: 4507-4522.

Johnston, M., D. Johnston \& A. Richardson. 2005. Digestive capabilities reflect the major food sources in three species of talitrid amphipods. Comp. Biochem. Physiol. B. 140: 251-257.

Kates, M. 1972. Techniques in Lipidology: isolation analysis and identification of lipids. Elsevier, Amsterdam, Holanda.

Lemos, M. 2002. Aspectos biológicos y bioensayos de toxicidad empleando Talorchestia margaritae (Amphipoda, Talitridae). Tesis de Licenciatura, Universidad Simón Bolívar, Caracas, Venezuela.

Mayzaud, P. \& R. Ackman. 1976. The occurrence and distribution of octadecapentaenoic Acid in a Natural Plankton Population. A Possible Food Chain Index. Lipids 11: 858-862.

Nelson, M., B. Mooney, P. Nichols \& C. Phleger. 2001. Lipids of Antarctic Ocean amphipods: food chain interactions and the occurrence of novel biomarkers. Mar. Chem. 73: 53-64.

Nelson, D. \& M. Cox. 2005. Lipids, p. 363-386. In D. Nelson \& M. Cox. Lehninger: Principles of Biochemistry. W.H. Freeman, Nueva York, EEUU.

Nyssen, F. 2005. Role of benthic amphipods in antarctic trophodynamics: a multidisciplinary study. Ph.D. Thesis, Universitié de Liège, Liège, Belgique.

Parrish, C., T. Abrajano, S. Budge, R. Helleur, E. Hudson, K. Pulchan \& C. Ramos. 2000. Lipid and Phenolic Biomarker in Marine Ecosystems: Analysis and Applications, p. 194-223. In P. Wangersky (ed.). The Handbook of Environmental Chemistry. Springer, Berlin, Alemania.

Poretti, T., M. Casset \& F. Momo. 2003. Composición química y dinámica poblacional de Hyalella curvispina en el arroyo las flores (cuenca del río Luján). Biol. Acuat. 20: 45-48.

Sánchez, L. 1985. Algunos aspectos biológicos de Talorchestia margaritae Stephensen, 1948 (Amphipoda: 
Talitridae), de la Isla de Margarita, Venezuela. Tesis de Licenciatura, Universidad de Oriente, Cumaná, Venezuela.

Scott, C., S. Falk-Petersen, B. Gulliksen, O. Lonne \& J. Sargent. 2001. Lipid indicators of the diet of the sympagic amphipod Gammarus wilkit zkii in Marginal Ice Zone and in open waters of Svalbard (Arctic). Polar Biol. 24: 572-576.

Stephensen, K. 1948. Amphipods from Curaçao, Bonaire, Aruba and Margarita. Studies on the Fauna of Curaçao, Bonaire, Aruba and Margarita. Stud. Fauna Curacao 9: 1-20.

Venables, B. 1981a. Oxygen consumption in a tropical beach amphipod, Talorchestia margaritae
Stephensen: Effects of size and temperature. Crustaceana 41: 89-94.

Venables, B. 1981b. Energy allocation for growth and metabolism in Talorchestia margaritae (Amphipoda, Talitridae). Crustaceana 41: 182-189.

Venables, B. 1981c. Aspects of the populations biology of Venezuelan beach amphipod, Talorchestia margaritae (Talitridae), including estimates of biomass and daily production, and respiration rates. Crustaceana 41: 271-285.

Zhou, X. \& G. Arthur. 1992. Improved procedures for the determination of lipid phosphorus by malachite green. J. Lipid Res. 33: 1233-1236. 